\title{
Obesity phenotype and intra-abdominal fat responses to regular aerobic exercise
}

\author{
Hiroyuki Sasai, $\mathrm{MS}^{1)}$, Yasutomi Katayama, $\mathrm{PhD}^{2)}$, Yoshio Nakata, $\mathrm{PhD}^{3)}$, \\ Hiroyuki Ohkubo, $\mathrm{MS}^{1)}$, Kiyoji Tanaka, $\mathrm{PhD}^{3)}$
}

1) Doctoral Program of Sports Medicine, Graduate School of Comprehensive Human

Sciences, University of Tsukuba

Address: 1-1-1 Tennodai, Tsukuba, Ibaraki 305-8574, Japan)

2) Faculty of Education, Kogakkan University

Address: 1704 Koudakujimoto-cho, Ise, Mie 516-8555, Japan

3) Graduate School of Comprehensive Human Sciences, University of Tsukuba

Address: 1-1-1 Tennodai, Tsukuba, Ibaraki 305-8574, Japan

Running head: Obesity phenotype and beneficial exercise effects

Article type: Original article

Address all correspondence and reprint requests to:

Hiroyuki Sasai, MS

Doctoral Program of Sports Medicine, Graduate School of Comprehensive

Human Sciences, University of Tsukuba

1-1-1 Tennodai, Tsukuba, Ibaraki 305-8574, Japan 


\section{Phone: +81-29-853-5600 (ext. 8365), Fax: +81-29-853-2986 \\ E-mail: sasai@stat.taiiku.tsukuba.ac.jp}

\section{Grant supports:}

1) The Tsukuba Advanced Research Alliance (TARA) Center at the University of Tsukuba (2004-2006, Tanaka Project)

2) The 21 st century COE program from the Japanese Ministry of Education, Culture, Sports, Science and Technology (2002-2006, Nishihira Project)

3) A grant-in-aid from the Japanese Ministry of Health, Labour and Welfare (2005-2007, Kita Project). 


\section{Structured abstract}

Aim: To examine the effects of obesity phenotype on abdominal fat responses to regular aerobic exercise.

Method: Fifty-seven obese men aged 34 to 70 years were divided into two groups

5 based on baseline intra-abdominal fat (IF) area measured by computerized tomography: moderate $\left(<200 \mathrm{~cm}^{2}\right)$ IF $(\mathrm{MIF} ; n=33$, age: $52.9 \pm 10.6$ [SD] yr, body mass index [BMI]: $\left.29.2 \pm 3.1 \mathrm{~kg} / \mathrm{m}^{2}\right)$ or high $\left(\geq 200 \mathrm{~cm}^{2}\right)$ IF $(\mathrm{HIF} ; n=24$, age: $53.5 \pm$ $9.5 \mathrm{yr}$, BMI: $30.3 \pm 3.1 \mathrm{~kg} / \mathrm{m}^{2}$ ). The 12 -week exercise program consisted primarily of aerobic exercise and met 3 days per week for 90 minutes per session. Weight, BMI,

10 body composition (by dual-energy X-ray absorptiometry), IF and subcutaneous fat (SF) areas were measured before and after the program.

Results: Regular aerobic exercise reduced weight by $2.3 \pm 2.2 \mathrm{~kg}$ in MIF and $3.2 \pm 3.0$

$\mathrm{kg}$ in HIF. HIF reduced significantly more IF area than MIF $\left(15.1 \pm 26.0 \mathrm{~cm}^{2}\right.$ in MIF and $43.3 \pm 41.9 \mathrm{~cm}^{2}$ in HIF), and the difference remained significant after adjusting for

15 baseline fat mass, weight change and total energy intake during the 12 -week aerobic exercise.

Conclusion: Exercise-induced IF reduction may be remarkably influenced by obesity phenotype.

20 Keywords: Intra-abdominal fat, regular exercise, obesity phenotype 


\section{Introduction}

It is well accepted that obesity is associated with a number of chronic diseases [1] such as hypertension, dyslipidemia and type-2 diabetes, and could be an important predictor for incident cardiovascular disease (CVD) and associated premature deaths

25 [2]. The prevalence of obesity has been increasing worldwide including in Asian countries such as Japan [3]. In Japan, despite the fact that only $2-3 \%$ has been characterized as obese [3], defined by the World Health Organization as a body mass index $(\mathrm{BMI}) \geq 30 \mathrm{~kg} / \mathrm{m}^{2}$, the prevalence of metabolic disorders are relatively high [4, 5]. This suggests that Japanese individuals are likely to develop CVD or other fatal

30 events at even mild levels of obesity. A possible mechanism underlying these relationships could be obesity phenotype as the Japanese population has been found to have greater intra-abdominal fat (IF) area than Caucasians of the same age, BMI, and waist [6]. It is well established that excess IF is strongly associated with CVD risk factors (e.g., hypertension [7] and type-2 diabetes [8, 9]). In 291 men (ages, $56.4 \pm$

3512.0 years), Kuk et al. [10] established a threshold of $200 \mathrm{~cm}^{2}$ above which risk of all-cause mortality was found to be increased.

Because obesity and IF accumulations are primarily induced by physical inactivity and excess energy intake, increased physical activity and dietary modification could be an effective way to reduce metabolic disorders $[11,12]$. Regular exercise may be more

40 effective at decreasing IF than dietary modification; regular exercise was found to decrease IF area without substantial weight change and preferentially reduce IF area compared with subcutaneous fat (SF) [13].

Although numerous investigations were conducted to determine the effects of regular aerobic exercise on IF levels [14], the results of these exercise studies vary 
45 considerably depending on characteristics of the participants at baseline [15]. It has been suggested that potential influencing factors include physiological, environmental and genetic attributes. Obesity phenotype is a possible factor influencing IF responses to regular aerobic exercise because of increased fat availability during a single bout of prolonged aerobic exercise in IF type obesity [16]. One study examined whether 50 obesity phenotype would affect the IF response to a weight-loss intervention in Japanese women [17]; however, there have been no studies investigating its effects on IF response to regular aerobic exercise alone.

The purpose of this investigation was to determine the effects of obesity phenotype on IF responses to regular exercise. We hypothesized that participants with 55 high levels of IF would reduce IF to a greater extent than those with moderate levels of IF. Clearing this mechanism will help establish better exercise programs to prevent incident CVD and premature death in the clinical setting. 


\section{Methods}

\section{Participants}

Participants aged 30 to 70 years were recruited through advertisements in local newspapers. Inclusion criteria were as follows: 1) without a history of CVD such as myocardial infarction and stroke, and 2) BMI of greater than $25 \mathrm{~kg} / \mathrm{m}^{2}$, according to the guidelines set by the Japanese Society for the Study of Obesity [18]. In total, 68 obese men who met the both criteria participated in this study.

Based on the computerized tomographic analysis, the participants were divided into two groups according to Kuk et al. [10]: moderate intra-abdominal fat (MIF) obesity (IF area $<200 \mathrm{~cm}^{2} ; n=40$ ) or high intra-abdominal fat (HIF) obesity (IF area $\geq$ $200 \mathrm{~cm}^{2} ; n=28$ ). Seven participants with MIF obesity and four with HIF obesity were unable to successfully complete the study. Hence, 33 men in the MIF obesity group, and 24 men in the IF obesity group completed the study requirements. Their baseline characteristics including prevalence of basal disease (i.e., hypertension, dyslipidemia or diabetes mellitus) and drug treatment, alcohol and smoking habit were included in Table 1.

Hypertension was defined as systolic blood pressure (BP) $\geq 140 \mathrm{mmHg}$, and/or

75 diastolic $\mathrm{BP} \geq 90 \mathrm{mmHg}$, and/or receiving current medication for this condition [19]. Dyslipidemia was defined as the presence of any one of the following four risks: 1) total cholesterol $\geq 220 \mathrm{mg} / \mathrm{dl}, 2$ ) low-density lipoprotein (LDL) cholesterol $\geq 140$ $\mathrm{mg} / \mathrm{dl}, 3$ ) high-density lipoprotein (HDL) cholesterol $<40 \mathrm{mg} / \mathrm{dl}, 4)$ triglycerides $\geq 150$ $\mathrm{mg} / \mathrm{dl}$, and/or receiving current medication for these conditions [20]. Fasting blood

80 glucose $(\mathrm{FBG}) \geq 126 \mathrm{mg} / \mathrm{dl}$ and/or receiving current medication for this condition was the criterion for diabetes mellitus [21]. An interview was conducted to ascertain the 
uses of antihypertensive and lipid medication, treatment for diabetes mellitus, alcohol (never, past-, or current drinker) habit, smoking (never, past-, or current smoker) habit, fasting status ( $\geq 8 \mathrm{~h})$, and any histories of CVD.

The purpose and design of the study were fully explained to each participant before they gave written informed consent. The research protocol was approved by the institutional review board at the University of Tsukuba.

\section{Exercise regimen}

All participants were involved in a 90-min combined exercise program consisting of aerobic exercise and light resistance training 3 days/week for 12 weeks. Each 90-minute exercise program began with 15-20 minutes of warm-up activities such as stretching and calisthenics. The exercise program was followed by $45-60$ minutes of brisk walking, mild jogging and/or aerobic dancing, and finished with 15-25 minutes

95 of recreational and cool-down activities. All exercise programs were performed under the supervision of several trained physical trainers at the research center at the University of Tsukuba. Participants were instructed to perform aerobic exercise at a level that raised their heart rates at or near the heart-rate level corresponding to their anaerobic threshold. Additionally, participants were recommended to perform other

100 kinds of exercise such as walking, aerobic dancing, and stationary cycling at or around their homes on days with no exercise program. Each participant recorded his mode and duration of this additional exercise on a printed form. To study the effects of exercise alone and eliminate the confounding effects of diet-induced weight loss, participants were counseled not to change their dietary habit throughout the study period. 


\section{Anthropometry and body composition measurements}

Height was measured to the nearest $0.1 \mathrm{~cm}$ using a wall-mounted stadiometer (YG-200; Yagami, Nagoya, Japan). Weight, percentage of fat mass (\%fat), whole-body fat mass (FM), and fat-free mass (FFM) were measured by dual-energy X-ray

110 absorptiometry using a Lunar DPX-NT densitometer (Lunar, Madison, WI, USA). Preand post-intervention measurements were conducted by the same examiner to minimize technical error. BMI was calculated as weight in kilograms divided by squared height in meters.

115 Measurement of maximal oxygen uptake

Maximal oxygen uptake $\left(\dot{\mathrm{V}}_{2} \max \right)$ for each participant was determined by a graded exercise test with a cycling ergometer (828E, Monark, Stockholm, Sweden). Following a 2-min warm up at 30 watts (W), the workload was increased every minute by $15 \mathrm{~W}$ until volitional exhaustion. During the test, ventilations and expiratory gases

120 were measured using an indirect calorimeter (Oxycon Alpha, Mijnhardt, Breda, Netherlands). The highest oxygen uptake achieved over 30 seconds was determined as $\dot{\mathrm{V}}_{2} \max$. The $\mathrm{V}_{2}$ max was analyzed in the method described by Tanaka et al. [22]. Anaerobic threshold (AT) was determined as a nonlinear increase in VE when plotted against $\dot{\mathrm{VO}}_{2}$ or a simultaneous breakpoint in $\dot{\mathrm{VE}} / \mathrm{V}_{2}$ and the partial pressure of oxygen 125 in end-tidal expired air [23].

\section{Abdominal fat area by computerized tomography}

The IF, SF and total fat (TF) areas (centimeters squared) were measured at the umbilicus level using computerized tomography (CT) scans (Somatom AR.C; Siemens, 
130 Erlangen, Germany) performed on participants in the supine position with their arms stretched above their head. Each participant was clothed only in a loose-fitting gown since restrictive clothing had been determined to alter the detection of fat distribution. Pre- and post-intervention measurements were conducted by the same technician. The IF and SF areas were calculated using a computer software program (FatScan;

135 N2system, Osaka, Japan) according to the method of Yoshizumi et al. [24]. We directly estimated visceral adiposity from the IF area. This measurement has been reported to have a high correlation with directly ascertained total visceral fat volume [25]. The intra-class correlation for repeated IF area determinations in our laboratory was 0.98 ( $n$ $=37)$.

140

\section{Measurement of cardiovascular risk factors}

Systolic and diastolic BP were measured by trained observers using a standard mercury sphygmomanometer (cuff size $14 \times 47 \mathrm{~cm}$ ) on the right arm of seated participants who had rested for at least 10 minutes. A blood sample was drawn from

145 each participant after an overnight $(\geq 8 \mathrm{~h})$ fast. Plasma concentrations of free fatty acid (FFA) were analyzed by colorimetric method. Serum total cholesterol and triglycerides were determined enzymatically, serum HDL cholesterol was measured by the heparin-manganese precipitation method. Serum LDL cholesterol was estimated according to the equation of Friedewald et al. [26]. FBG was assayed by a glucose 150 oxidase method.

\section{Energy intake assessments}

Total energy intake (TEI) in kilocalories and macronutrient (carbohydrates, 
protein, and fat) balances before and during a 2-week period (weeks 9 and 10) of the

155 exercise program were assessed by 3-day weighed dietary records (3-day WDR) and dietary recall interviews for each participant performed by skilled dieticians. We explained how to complete the 3-day WDR in detail before taking measurements. The participants learned to use a digital cooking scale for weighing food. The dietitians collected the recorded sheets from the 3-day WDR and codified the food items and

160 food weights. Thereafter, the dieticians interviewed each participant to elicit more information about their food intake for the 3-day period. Based on the Standard Tables of Food Composition in Japan fourth revised edition [27], TEI along with carbohydrate, fat, and protein intakes were calculated.

\section{$165 \quad$ Energy expenditure assessments}

The total energy expenditure (TEE) and physical activity energy expenditure (PAEE) were assessed by a uniaxial accelerometer (Lifecorder; Suzuken Co. Ltd., Nagoya, Japan). Measurements of TEE using the accelerometer were validated against doubly-labeled water method [28] and metabolic chamber method [29], both of which

170 were considered as free-living and laboratory based gold standards for TEE measurements, respectively. The accelerometer can assess two types of activity-related energy expenditure: energy expenditure of activities $\left(E_{\mathrm{Act}}\right)$ indicating $\geq 1.8$ metabolic equivalents (METs) and energy expenditure of minor activities (EE $\left.E_{\text {minorAct }}\right)$ indicating $<1.8$ METs [30]. In this study, the former was considered as PAEE. The TEE was

175 calculated from the sum of the basal metabolic rate (BMR), diet-induced thermogenesis $(10 \% \mathrm{TEE}), \mathrm{EE}_{\mathrm{Act}}$ and $\mathrm{EE}_{\text {minorAct. }}$ The BMR was estimated from sex, age, weight and height using a standard Japanese formula [31]. Detailed descriptions of the 
accelerometer have been published elsewhere [29]. Participants wore the accelerometer regularly (except while sleeping or bathing), for a 2-week period prior

180 to the 12-week intervention and during a 2-week period during the intervention (weeks 9 and 10). To eliminate reporting bias, no correction was used for those activities (swimming, weight lifting, cycling, etc), which can not be captured by the accelerometers.

\section{$185 \quad$ Statistical analysis}

All results were shown as mean \pm standard deviation. To compare change in each item between groups, a two-way repeated measures analysis of variance (time $\times$ group) was applied. An unpaired Student's $t$ test or a chi-squared test was used to compare variables between the groups at baseline. A paired Student's $t$ test was used to

190 determine the significance of differences between pre- and post-intervention data. An analysis of covariance (ANCOVA) was used, with absolute changes acting as dependent variables, and baseline FM, weight changes and TEI during 12-week regular exercise acting as the covariates. The mean values obtained from the ANCOVAs were used to interpret group differences. A $p$ value of less than 0.05 was considered

195 statistically significant. Data were analyzed with the Statistical Analysis System (version 9.01; SAS Institute Inc., Cary, NC, USA). 


\section{Results}

Attendance at exercise sessions was $87 \%$ (range, 54\% to $100 \%$ ) for MIF obesity group, and $85 \%$ (range $41 \%$ to $100 \%$ ) for HIF obesity group. Table 1 contains baseline

200 characteristics of study participants and their responses to regular aerobic exercise. There were no differences at baseline between groups for age, prevalence of basal disease, medication uses except for hypertension, alcohol and smoking habit, BMI and \%fat, while height, weight, FM and FFM were significantly higher in HIF obesity than those values in MIF obesity. $\dot{\mathrm{VO}}_{2}$ max was significantly lower in HIF obesity than

205 that in MIF obesity. Significant reductions were observed in weight, BMI, FM and \% fat within each group. Significant increases in $\mathrm{VO}_{2} \max$, not in AT and heart rate at AT, were detected within each group. ANOVA revealed no significant interactions ( $p$ $=0.20-0.95)$ for all variables between MIF and HIF obesity.

The values for energy intake and expenditure during 2-week periods are shown in

210 Table 2. There were no baseline differences for TEI and macronutrient balances between the two groups. For both groups, although TEI and macronutrient balance remained unchanged, TEE and PAEE significantly increased during the study period. However, no significant interactions $(p=0.19-0.92)$ were detected among the groups in the absolute change of any variable.

215 Table 3 shows comparisons between the groups in baseline abdominal fat, other cardiovascular risk factors and its responses to regular aerobic exercise. Although there was no significant difference in SF area between the groups at baseline, participants with HIF obesity exhibited greater IF and TF areas than that in MIF obesity. Although regular aerobic exercise significantly reduced all abdominal fat 220 variables in both groups, significantly greater reductions in IF and TF areas were 
observed in participants with HIF obesity compared to those with MIF obesity. Baseline levels of FFA and LDL cholesterol were significantly higher in HIF group than MIF group. Total and LDL cholesterol, and triglycerides in both groups, and diastolic BP and HDL cholesterol in the MIF group were significantly improved after

225 the regular exercise despite the fact that the interactions did not reach statistical significance in these cardiovascular risk factors.

Figure 1 illustrates abdominal fat responses to regular aerobic exercise after adjusting for possible confounders (baseline FM, weight change and TEI during regular aerobic exercise), which represents the overall energy balance of the 12 -week 230 intervention. ANCOVAs demonstrated that reductions in IF and TF areas were significantly greater in HIF obesity compared to those in MIF obesity after controlling for covariates. 


\section{Discussion}

The present study was conducted to determine the effects of obesity phenotype on

235 IF response to regular exercise. We hypothesized participants classified as HIF obesity would reduce IF, to a greater extent, than those classified as MIF obesity. It was found that when baseline FM, weight change and TEI were controlled for in the analyses, those with HIF obesity exhibited greater reductions in IF and TF areas than those with MIF obesity.

These phenomena might be explained by enhanced lipolytic properties of IF tissues. It seems that IF tissue has higher lipolytic responses to catecholamine compared to SF tissue [31], which markedly increases during endurance exercise in an intensity-dependent manner [32]. On the other hand, IF tissue has lower antilipolytic responses to insulin compared to SF tissue [33], which gradually decreases during

245 prolonged exercise. A previous report [16] indicated higher lipid concentrations in HIF obesity than MIF obesity in men. However, whole-body fat oxidation did not differ between HIF and MIF obesity phenotypes. In the present study, we also demonstrated that whole-body fat loss did not differ significantly between HIF and MIF obesity phenotypes. In this respect, our results were consistent with the previous study. This

250 indicated that fat availability from IF tissue during exercise might be higher in HIF obesity than MIF obesity.

Increased growth hormone $(\mathrm{GH})$ concentrations during exercise could also be proposed as a potential mechanism. GH is a strong lipolytic hormone that significantly increases during endurance exercise [34]. In an in-vitro study using rat adipocytes [35],

255 GH additions stimulated FFA release from both IF and SF adipocytes, and its effects were more prominent in IF adipocytes compared to SF adipocytes. In a recent human 
study [36], GH administration induced greater reductions of IF tissue than SF tissue. Obesity is associated with a decrease in insulin-like growth factor-I and deficits in GH secretion. This is particularly apparent in individuals with accumulation of visceral

260 adiposity [37]. Increases in FFA, a hallmark of visceral adiposity, are known to decrease GH secretion. Svensson et al. [38] tested the effectiveness of GH therapy in men with visceral obesity. They observed a striking reduction in IF tissue (-18\%) along with an increase in lean muscle mass $(+2 \%)$. The effect of GH administration on fat distribution was more prominent in GH-deficient adults than in healthy obese men;

265 similarly, central obesity known to decrease GH secretion may be sensitive to $\mathrm{GH}$-induced lipolysis by aerobic exercise. However, it is unknown whether GH responses to acute aerobic exercise differ between HIF or MIF obesity phenotypes. In addition, since we did not measure participant's blood concentration of GH during exercise and at rest, we were unable to address this issue.

Another possible mechanism is the influence of BMR on abdominal fat tissue. Some investigators have reported that IF tissue was positively related to BMR in middle-aged obese adults. Similar, yet inconsistent, results have been found for women [39]. Most studies in men using CT as well as anthropometric variables such as waist and waist to hip ratio showed comparable results, indicating that IF tissue is an

275 important determinant of BMR [40]. With this in mind, we could speculate that increased BMR during exercise and at rest induced differences in total energy expenditure between HIF and MIF obesity phenotypes. The uniaxial accelerometer in our study estimated BMR using age, height, and weight as well as sex, but did not consider the altered fat oxidation rate associated with visceral adiposity. Therefore, the 280 accelerometer might have underestimated TEE in HIF obesity. This inaccurate 
measurement of BMR may have created greater IF reductions for the HIF obesity phenotype. It has been reported that fat oxidation rate during resting conditions was also related to obesity phenotype [36]. Because fat oxidation rate is related to plasma FFA concentrations, an enhanced release of the metabolite from IF adipose tissue may

285 increase the fat oxidation rate in participants with HIF. Indeed, our HIF participants showed significantly higher resting FFA concentrations than MIF groups. Overall, these potential mechanisms may have interactively affected the differences in IF response to regular aerobic exercise between the two groups.

Several intervention studies have examined the influences of obesity phenotypes 290 on fat distributions in response to lifestyle modification such as diet alone or in combination with exercise. Leenen et al. [41] demonstrated that the amount of IF loss during weight loss was related to the initial amount of IF accumulation. This suggests that obese people with an initial abundance of IF preferentially lose IF compared to those people with less IF. Additionally, Okura et al. [11] showed that IF type ( $\geq 110$ $295 \mathrm{~cm}^{2}$ ) obese women had significantly greater improvements in body fat distribution compared to SF type $\left(<110 \mathrm{~cm}^{2}\right)$ obese women. Therefore, our observation that body fat distribution can be reduced to a great extent by regular exercise in those with high levels of IF is consistent with several previous studies.

One strength of our study was our ability to determine that the negative energy 300 balance in both phenotypes was caused by increased energy expenditure during the 12-week exercise intervention. Energy intake in the present study was assessed by a 3-day WDR which has often been used as the criterion method for exterminating energy intake. Moreover, there were no significant changes in energy intake during the intervention in both obesity phenotypes. Therefore, it is likely that beneficial effects 
305 on abdominal fat tissues were mainly induced by increased physical activities alone. Previous studies solely determined the effects of obesity phenotypes on abdominal fat tissues in response to dietary modification with or without regular exercise $[11,17]$.

Our study has some limitations. First, the exercise mode was not rigorously controlled (i.e., intensity and frequency). Exercise intensity has been reported to affect

310 IF fat availability due to increased catecholamine [42]. With regard to variation of exercise frequency, it is known that the amount of physical activity (energy expenditure) is more important for reducing abdominal fat than the frequency of exercise [13]. However, there was no significant difference in weight change which would indicate differential energy balance accumulations over the intervention period.

315 Moreover, our data were analyzed using ANCOVA with weight change as a covariate, and the influence of differential energy expenditure on IF tissue was excluded in the results of this study. Second, since we divided participants into two obesity phenotypes based on their initial levels of IF area, their responses to regular aerobic exercise might be partly affected by so-called "regression to the mean" effects. We are unlikely to be

320 able to eliminate these effects, so the values of IF area reduction could be overestimated. It is possible that these limitations may have interfered with the effects of obesity phenotype on IF responses to the regular aerobic exercise.

In summary, as the amount of SF lost in response to $2-3 \mathrm{~kg}$ of exercise-induced weight loss did not differ between MIF and HIF obesity phenotypes, the improvement 325 in IF area was significantly greater for those with HIF obesity than for those with MIF obesity. These results suggest that IF responses to regular exercise predominantly depend on the obesity phenotype. In clinical settings for obesity treatments through exercise, clinical care personnel should consider that individuals with accumulated IF 
area may exhibit a more sensitive IF response to regular exercise compared with MIF

330 obesity, and therefore, they need to deliver appropriate exercise prescriptions for those with both MIF and HIF obesity phenotypes to improve IF accumulation and prevent CVD events.

335 5. Acknowledgements

This work was supported in part by the Tsukuba Advanced Research Alliance (TARA) Center at the University of Tsukuba (2004-2006, Tanaka Project), the 21 st century COE program from the Japanese Ministry of Education, Culture, Sports, Science and Technology (2002-2006, Nishihira Project), and a grant-in-aid from the 340 Japanese Ministry of Health, Labour and Welfare (2005-2007, Kita Project). 


\section{References}

1. Caterson I, Hubbard V, Bray G, Grunstein R, Hansen B, Hong Y, et al. Prevention conference VII. Obesity, a worldwide epidemic related to heart disease and stroke group III: worldwide comorbidities of obesity. Circulation 2004; 110: 476-483.

345 2. Klein S, Burke L, Bray G, Blair S, Allison D, Pi-Sunyer X, et al. Clinical implications of obesity with specific focus on cardiovascular disease. A statement for professionals from the American Heart Association on nutrition, physical activity, and metabolism. Circulation 2004; 110: 2952-2967.

3. Yoshiike N, Matsumura Y, Zaman MM, Yamaguchi M. Descriptive epidemiology of body mass index in Japanese adults in a representative sample from the National Nutrition Survey 1990-1994. Int J Obes Relat Metab Disord 1998; 22: 684-687.

4. Kuzuya T. Prevalence of diabetes mellitus in Japan compiled from literature. Diabetes Res Clin Pract 1994; 24: S15-21.

5. Sakata K, Labarthe DR. Changes in cardiovascular disease risk factors in three Japanese national surveys 1971-1990. J Epidemiol 1996; 6: 93-107.

6. Kadowaki T, Sekikawa A, Murata K, Maegawa H, Takamiya T, Okamura T, et al. Japanese men have larger areas of visceral adipose tissue than Caucasian men in the same levels of waist circumference in a population-based study. Int J Obes 2006; 30: 1163-1165.

360 7. Hayashi T, Boyko EJ, Leonetti DL, McNeely MJ, Newell-Morris L, Kahn SE, Fujimoto WY. Visceral adiposity is an independent predictor of incident hypertension in Japanese Americans. Ann Intern Med 2004; 140: 992-1000.

8. Hayashi T, Boyko EJ, Leonetti DL, McNeely MJ, Newell-Morris L, Kahn SE, Fujimoto WY. Visceral adiposity and the risk of impaired glucose tolerance: a 
prospective study among Japanese Americans. Diabetes Care 2003; 26: 650-655.

9. Boyko EJ, Fujimoto WY, Leonetti DL, Newell-Morris L. Visceral adiposity and risk of type 2 diabetes: a prospective study among Japanese Americans. Diabetes Care 2000; 23: 465-471.

10. Kuk JL, Katzmarzyk PT, Nichaman MZ, Church TS, Blair SN, Ross R. Visceral fat is an independent predictor of all-cause mortality in men. Obesity 2006; 14 : $336-341$.

11. Okura T, Tanaka K, Nakanishi T, Lee DJ, Nakata Y, Wee SW, et al. Effects of obesity phenotype on coronary heart disease risk factors in response to weight loss. Obes Res 2002; 10: 757-766.

375 12. Tanaka K, Okura T, Shigematsu R, Nakata Y, Lee DJ, Wee SW, et al. Target value of intraabdominal fat area for improving coronary heart disease risk factors. Obes Res 2004; 12: 695-703.

13. Ross R, Dagnone D, Jones PJ, Smith H, Paddags A, Hudson R, et al. Reduction in obesity and related comorbid conditions after diet-induced weight loss or exercise-induced weight loss in men. A randomized, controlled trial. Ann Intern Med 2000; 133: 92-103.

14. Kay SJ, Fiatarone Singh MA. The influence of physical activity on abdominal fat: a systematic review of the literature. Obes Rev 2006; 7: 183-200.

15. Crouse SF, O'Brien BC, Grandjean PW, Lowe RC, Rohack JJ, Green JS, et al. Training intensity, blood lipids, and apolipoproteins in men with high cholesterol. J Appl Physiol 1997; 82: 270-277.

16. Numao S, Hayashi Y, Katayama Y, Matsuo T, Tomita T, Ohkawara K, et al. Effects of obesity phenotype on fat metabolism in obese men during endurance exercise. 
Int J Obes 2006; 30: 1189-1196.

390 17. Okura T, Nakata Y, Lee DJ, Ohkawara K, Tanaka K. Effects of aerobic exercise and obesity phenotype on abdominal fat reduction in response to weight loss. Int $\mathbf{J}$ Obes 2005; 29: 1259-1266.

18. Examination Committee of Criteria for 'Obesity Disease' in Japan; Japan Society for the Study of Obesity. New criteria for 'obesity disease' in Japan. Circ J 2002; 66: 987-992.

19. Japanese Society of Hypertension Guidelines Subcommittee for the Management of Hypertension. Guidelines for the management of hypertension for general practitioners. Hypertens Res 2001; 24: 613-634.

20. Hata Y, Mabuchi H, Saito Y, Itakura H, Egusa G, Ito H et al., Working Committee on JAS Guideline for Diagnosis and Treatment of Hyperlipidemias. Report of the Japan Atherosclerosis Society (JAS): Guideline for Diagnosis and Treatment of Hyperlipidemia in Japanese Adults. J Atheroscler Thromb 2002; 9: 1-27.

21. Kuzuya T, Nakagawa S, Satoh J, Kanazawa Y, Iwamoto Y, Kobayashi M, et al., The Committee of the Japan Diabetes Society on the diagnostic criteria of diabetes mellitus. Report of the Committee on the classification and diagnostic criteria of diabetes mellitus. Diabetes Res Clin Pract 2002; 55: 65-85.

22. Tanaka K, Takeshima N, Kato T, Niihata S, Ueda K. Critical determinants of endurance performance in middle-aged and elderly endurance runners with heterogeneous training habits. Eur J Appl Physiol Occup Physiol 1990; 59: $410 \quad 443-449$.

23. Beaver WL, Wasserman K, Whipp BJ. A new method for detecting anaerobic threshold by gas exchange. J Appl Physiol 1986; 60: 2020-2027. 
24. Yoshizumi T, Nakamura T, Yamane M, Islam AH, Menju M, Yamasaki K, et al. Abdominal fat: standardized technique for measurement at CT. Radiology 1999; 211: 283-286.

25. Borkan GA, Gerzof SG, Robbins AH, Hults DE, Silbert CK, Silbert JE. Assessment of abdominal fat content by computed tomography. Am J Clin Nutr 1982; 36 : $172-177$.

26. Friedewald WT, Levy RI, Fredrickson DS. Estimation of the concentration of low-density lipoprotein cholesterol in plasma, without use of the reparative ultracentrifuge. Clin Chem 1972; 18: 499-502.

27. Science and Technology Agency. Standard tables of food composition in Japan. 4th revised ed. Tokyo: Printing Bureau. Ministry of Finance; 1982 [in Japanese].

28. Rafamantanantsoa HH, Ebine N, Yoshioka M, Higuchi H, Yoshitake Y, Tanaka H, et al. Validation of three alternative methods to measure total energy expenditure against the doubly labeled water method for older Japanese men. J Nutr Sci Vitaminol 2002; 48: 517-523.

29. Kumahara H, Schutz Y, Ayabe M, Yoshioka M, Yoshitake Y, Shindo M, et al. The use of uniaxial accelerometry for the assessment of physical-activity-related energy expenditure: a validation study against whole-body indirect calorimetry. $\mathrm{Br}$ J Nutr 2004; 91: 235-243.

30. Health Promotion and Nutrition Division, Health Service Bureau, Ministry of Health and Welfare (1996) Recommended Dietary Allowances for the Japanese, 5th revision pp. 20-24. Tokyo: Dai-ichi shuppan Co. Ltd.

435 31. Hellmér J, Marcus C, Sonnenfeld T, Arner P. Mechanisms for differences in lipolysis between human subcutaneous and omental fat cells. J Clin Endocrinol 
Metab 1992; 75: 15-20.

32. Romijn JA, Coyle EF, Sidossis LS, Gastaldelli A, Horowitz JF, Endert E, et al. Regulation of endogenous fat and carbohydrate metabolism in relation to exercise intensity and duration. Am J Physiol 1993; 265: E380-E391.

33. Richelsen B, Pedersen SB, Møller-Pedersen T, Bak JF. Regional differences in triglyceride breakdown in human adipose tissue: effects of catecholamines, insulin, and prostaglandin E2. Metabolism 1991; 40: 990-996.

34. Stokes K. Growth hormone responses to sub-maximal and sprint exercise. Growth Horm IGF Res 2003; 13: 225-238.

35. Itoh Y, Shirai K, Miyashita Y, Watanabe H, Tomioka H, Irie M. Preferential lipolysis in rat visceral adipose tissues by growth hormone. Endocrinol Metab 1997; 4: 61-67.

36. Pasarica M, Zachwieja JJ, Dejonge L, Redman S, Smith SR. Effect of growth hormone on body composition and visceral adiposity in middle-aged men with visceral obesity. J Clin Endocrinol Metab 2007; 92: 4265-4270.

37. Vahl N, Jorgensen JO, Skjaerbaek C, Veldhuis JD, Orskov H, Christiansen JS. Abdominal adiposity rather than age and sex predicts mass and regularity of GH secretion in healthy adults. Am J Physiol 1997; 272: E1108-1116.

455 38. Svensson J, Bengtsson BA, Taskinen MR, Wiklund O, Johannsson G. A nine-month, placebo-controlled study of the effects of growth hormone treatment on lipoproteins and LDL size in abdominally obese men. Growth Horm IGF Res 2000; 10: $118-126$.

39. Nicklas BJ, Goldberg AP, Bunyard LB, Poehlman ET. Visceral adiposity is associated with increased lipid oxidation in obese, postmenopausal women. Am J 
Clin Nutr 1995; 62: 918-922.

40. Armellini F, Zamboni M, Mino A, Bissoli L, Micciolo R, Bosello O.

Postabsorptive resting metabolic rate and thermic effect of food in relation to body composition and adipose tissue distribution. Metabolism 2000; 49: 6-10.

465 41. Leenen R, van der Kooy K, Deurenberg P, Seidell JC, Weststrate JA, Schouten FJ, et al. Visceral fat accumulation in obese subjects: relation to energy expenditure and response to weight loss. Am J Physiol 1992; 263: E913-E919.

42. Numao S, Hayashi Y, Katayama Y, Matsuo T, Tomita T, Ohkawara K, et al. Plasma fat concentration increases in visceral fat obese men during high-intensity endurance exercise. Obes Res Clin Pract 2007; 1: 273-279. 
Tables \& Figure

Table 1. Baseline physical characteristics and responses to regular aerobic exercise.

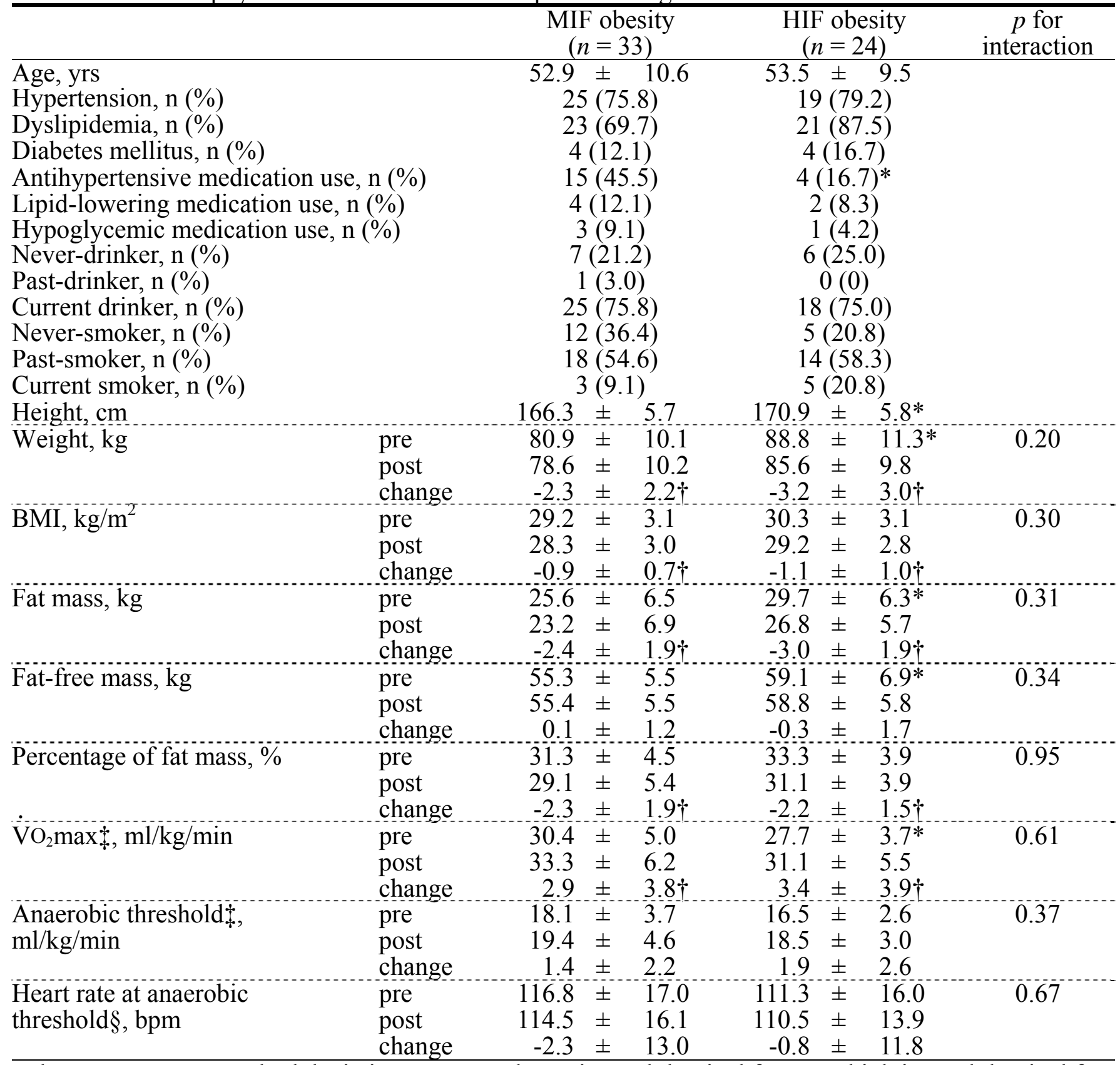

Values are mean \pm standard deviation, MIF: moderate intra-abdominal fat, HIF: high intra-abdominal fat, BMI: body mass index, $\mathrm{VO}_{2}$ max: maximal oxygen uptake, * significant differences between groups at pre by unpaired $t$ test or chi-squared test. $\dagger$ significant changes within group by paired $t$ test. Hypertension was defined as systolic blood pressure $\geq 140 \mathrm{mmHg}$, and/or diastolic blood pressure $\geq 90 \mathrm{mmHg}$, and/or receiving antihypertensive medication. Dyslipidemia was defined as the presence of any one of the following four risks: 1) total cholesterol $\geq 220 \mathrm{mg} / \mathrm{dl}$, 2) low-density lipoprotein cholesterol $\geq 140 \mathrm{mg} / \mathrm{dl}$, 3) high-density lipoprotein cholesterol $<40 \mathrm{mg} / \mathrm{dl}$, 4) triglycerides $\geq 150 \mathrm{mg} / \mathrm{dl}$, and/or receiving lipid-lowering medication. Fasting blood glucose $\geq 126 \mathrm{mg} / \mathrm{dl}$ and/or receiving hypoglycemic medication was the criterion for diabetes mellitus. $\$ 32$ data in SF obesity and 23 in IF obesity were available. $\S 31$ data in SF obesity and 23 in IF obesity were available. 
Table 2. Changes in energy intake and expenditure during the 12-week exercise intervention.

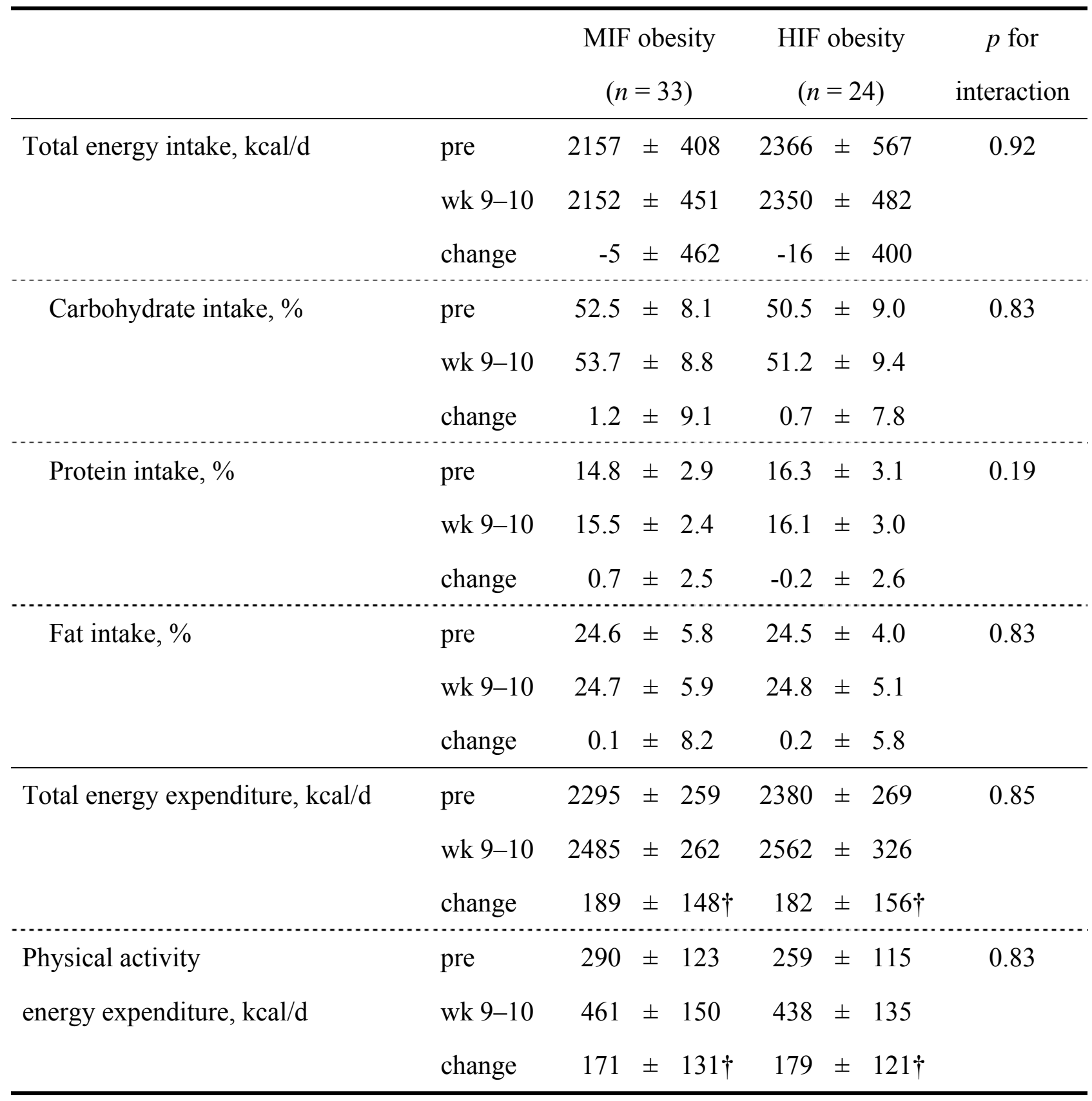

Values are mean \pm standard deviation, MIF: moderate intra-abdominal fat, HIF: high intra-abdominal fat, *significant difference between groups at pre by unpaired $t$ test. $\uparrow$ significant changes within group by paired $t$ test. 
Table 3. Abdominal fat and other cardiovascular risk factors at baseline and responses to regular aerobic exercise.

\begin{tabular}{|c|c|c|c|c|}
\hline & & $\begin{array}{l}\text { MIF obesity } \\
(n=33)\end{array}$ & $\begin{array}{l}\text { HIF obesity } \\
(n=24)\end{array}$ & $\begin{array}{c}p \text { for } \\
\text { interaction }\end{array}$ \\
\hline \multirow{3}{*}{ Intra-abdominal fat area, $\mathrm{cm}^{2}$} & pre & $149.7 \pm 35.4$ & $242.4 \pm 34.4^{*}$ & $<0.01$ \\
\hline & post & $134.6 \pm 43.1$ & $199.1 \pm 39.7$ & \\
\hline & change & $-15.1 \pm 26.0 \dagger$ & $-43.3 \pm 41.9 \dagger$ & \\
\hline \multirow[t]{3}{*}{ Subcutaneous fat area, $\mathrm{cm}^{2}$} & pre & $211.6 \pm 85.7$ & $235.0 \pm 80.3$ & 0.04 \\
\hline & post & $195.1 \pm 88.1$ & $203.0 \pm 68.5$ & \\
\hline & change & $-16.5 \pm 23.3 \dagger$ & $-32.1 \pm 32.9 \dagger$ & \\
\hline \multirow[t]{3}{*}{ Total fat area, $\mathrm{cm}^{2}$} & pre & $361.3 \pm 102.9$ & $477.4 \pm 98.8^{*}$ & $<0.01$ \\
\hline & post & $329.7 \pm 107.4$ & $402.1 \pm 74.8$ & \\
\hline & change & $-31.6 \pm 43.3 t$ & $-75.4 \pm 67.7 \dagger$ & \\
\hline \multirow[t]{3}{*}{ Systolic BP, mmHg } & pre & $140.1 \pm 14.9$ & $143.2 \pm 18.0$ & 0.58 \\
\hline & post & $137.4 \pm 13.9$ & $138.9 \pm 15.2$ & \\
\hline & change & $-2.7 \pm 10.3$ & $-4.3 \pm 10.6$ & \\
\hline \multirow{3}{*}{ Diastolic $\mathrm{BP}, \mathrm{mmHg}$} & pre & $92.1 \pm 10.2$ & $93.9 \pm 13.1$ & 0.40 \\
\hline & post & $89.5 \pm 10.4$ & $92.9 \pm 12.1$ & \\
\hline & change & $-2.6 \pm 7.1 \dagger$ & $-1.0 \pm 7.1$ & \\
\hline \multirow[t]{3}{*}{ Free fatty acid, mEq/1 } & pre & $0.59 \pm 0.21$ & $0.73 \pm 0.24 *$ & 0.30 \\
\hline & post & $0.56 \pm 0.19$ & $0.63 \pm 0.25$ & \\
\hline & change & $-0.03 \pm 0.24$ & $-0.10 \pm 0.22 \dagger$ & \\
\hline \multirow[t]{3}{*}{ Total cholesterol, mg/dl } & pre & $218.0 \pm 32.4$ & $232.4 \pm 30.2$ & 0.38 \\
\hline & post & $203.3 \pm 28.2$ & $212.0 \pm 33.4$ & \\
\hline & change & $-14.7 \pm 20.1 \dagger$ & $-20.4 \pm 26.7 \dagger$ & \\
\hline \multirow[t]{3}{*}{ LDL cholesterol, mg/dl } & pre & $130.1 \pm 30.9$ & $147.5 \pm 30.4^{*}$ & 0.39 \\
\hline & post & $120.7 \pm 23.3$ & $133.5 \pm 31.7$ & \\
\hline & change & $-9.5 \pm 15.0 \dagger$ & $-13.9 \pm 22.3 \dagger$ & \\
\hline \multirow[t]{3}{*}{ HDL cholesterol, mg/dl } & pre & $57.0 \pm 16.2$ & $50.6 \pm 9.2$ & 0.83 \\
\hline & post & $59.0 \pm 18.5$ & $52.3 \pm 9.3$ & \\
\hline & change & $2.0 \pm 5.1 \dagger$ & $1.7 \pm 6.1$ & \\
\hline \multirow[t]{3}{*}{ Triglycerides, $\mathrm{mg} / \mathrm{dl}$} & pre & $164.1 \pm 100.8$ & $172.0 \pm 74.3$ & 0.80 \\
\hline & post & $118.3 \pm 60.9$ & $131.1 \pm 48.0$ & \\
\hline & change & $-45.7 \pm 73.0 \dagger$ & $-40.8 \pm 61.2 \dagger$ & \\
\hline \multirow[t]{3}{*}{ Fasting blood glucose, $\mathrm{mg} / \mathrm{d} \mathrm{l}$} & pre & $98.7 \pm 13.9$ & $103.0 \pm 17.1$ & 0.33 \\
\hline & post & $103.2 \pm 27.1$ & $103.2 \pm 13.3$ & \\
\hline & change & $4.5 \pm 16.5$ & $0.2 \pm 15.1$ & \\
\hline
\end{tabular}

Values are mean \pm standard deviation, MIF: moderate intra-abdominal fat, HIF: high intra-abdominal fat, BP: blood pressure, LDL: low-density lipoprotein, HDL: high-density lipoprotein, *significant difference between groups at pre by unpaired $t$ test. $\uparrow$ significant changes within group by paired $t$ test. Thirty and 23 data from blood samples were available in MIF and HIF groups, respectively. 


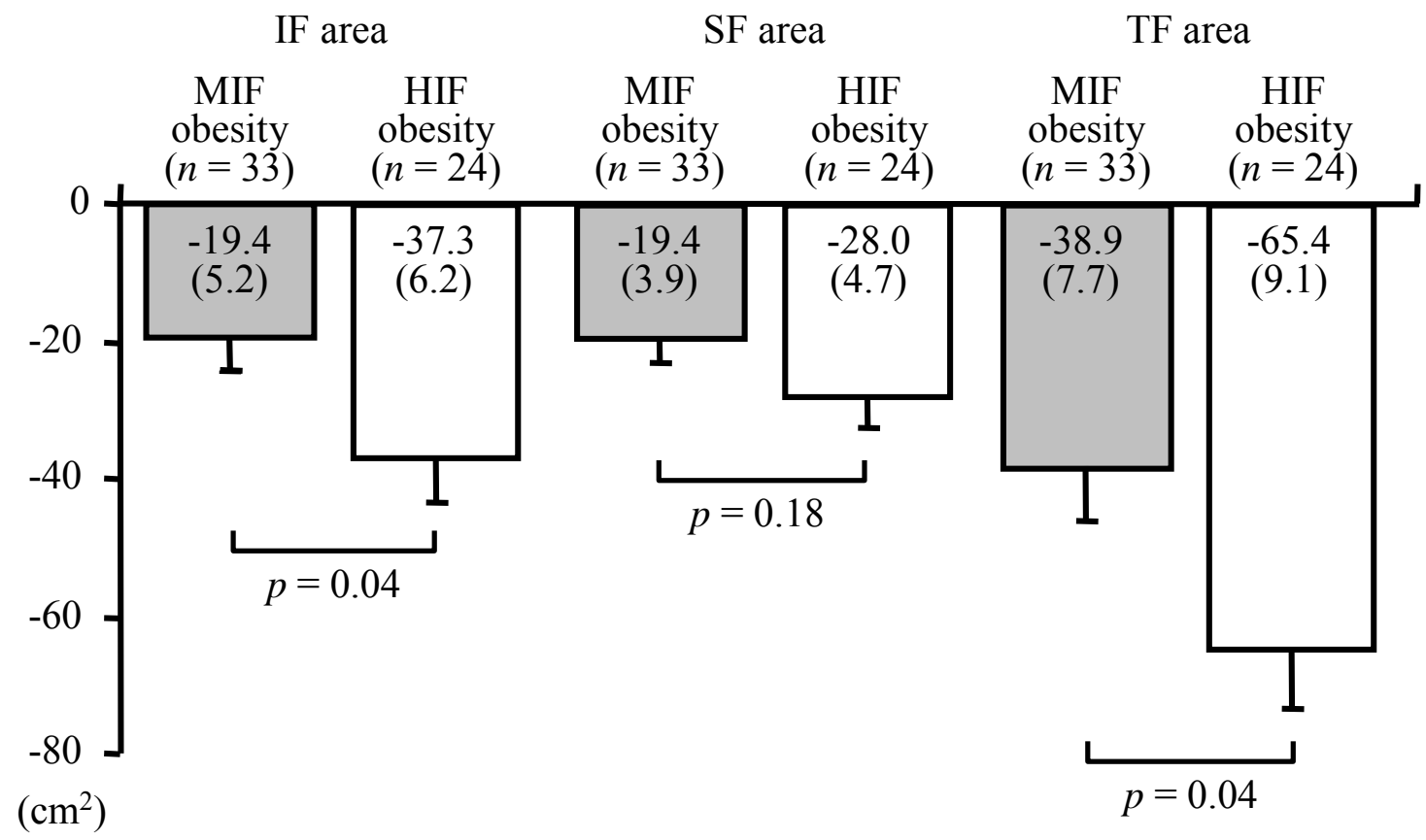

Figure 1. Abdominal fat responses to regular aerobic exercise after adjusting for baseline fat mass, weight changes and total energy intake during regular aerobic exercise. Values are expressed as mean (standard error). IF: intra-abdominal fat, SF: subcutaneous fat, TF: total fat (IF plus SF areas), MIF: moderate intra-abdominal fat, HIF: high intra-abdominal fat. 